\title{
BMJ Open Prevalence, incidence, and risk factors for myopia among urban and rural children in southern China: protocol for a school-based cohort study
}

Xin Chen, ${ }^{1,2}$ Guofang Ye, ${ }^{1}$ Yuxin Zhong, ${ }^{1}$ Ling Jin,${ }^{3}$ Xiaoling Liang, ${ }^{1}$ Yangfa Zeng, ${ }^{1}$ Yingfeng Zheng (D) , ${ }^{1,2}$ Morgan lan, ${ }^{1,4}$ Yizhi Liu, ${ }^{1,2}$

To cite: Chen $X$, Ye G, Zhong $Y$, et al. Prevalence, incidence, and risk factors for myopia among urban and rural children in southern China: protocol for a school-based cohort study. BMJ Open 2021;11:e049846. doi:10.1136/ bmjopen-2021-049846

- Prepublication history for this paper is available online. To view these files, please visit the journal online (http://dx.doi. org/10.1136/bmjopen-2021049846).

$\mathrm{YZ}$ and $\mathrm{YZ}$ contributed equally.

$\mathrm{XC}$ and GY are joint first authors.

Received 03 February 2021 Accepted 21 October 2021

\section{ABSTRACT}

Introduction Myopia is the common cause of reduced uncorrected visual acuity among school-age children. It is more prevalent in urban than in rural areas. Although many myopia studies have focused on the effect of urbanisation, it remains unclear how visual experience in urban regions could affect childhood myopia. This study aims to investigate the incidence and prevalence of myopia among school-age children in urban and rural settings, thereby identifying the environmental factors that affect the onset and progression of myopia.

Methods and analysis A school-based cohort study will be conducted. We will enroll all first-grade students from an urban (10 primary schools) and a rural (10 primary schools) regions of Zhaoqing city, China. Over 3-year follow-up period, students will receive detailed eye examinations annually and complete questionnaires about living habits and environment. In a 5\% random subsample of the cohort, physical activity, light intensity and eyetracking data will be obtained using wearable devices, and high-resolution macular images will be obtained by optical coherence tomography (OCT). The primary outcome is incident myopia, defined as myopia (spherical equivalent refractive of at least $-0.5 \mathrm{D}$ ) detected during follow-up among those without myopia at baseline.

Ethics and dissemination Ethics approval was obtained from the ethics committee of the Zhongshan Ophthalmic Center (number: 2019KYPJ171). Study findings will be published in a peer-reviewed journal.

Trial registration number NCT04219228.

\section{INTRODUCTION}

Myopia is the major cause of reduced uncorrected visual acuity (UCVA) among children and adolescents, particularly in the urban areas of East Asia. ${ }^{1}$ Studies from Australia, ${ }^{2}$ mainland $\mathrm{China}^{3{ }^{4}}$ and other regions ${ }^{5}$ have shown a consistent association between geography and myopia. Based on the severe myopia epidemic, there is great interest in understanding the risk factors for myopia children, especially those who may be suitable for intervention. A systematic and comprehensive analysis of the rural-urban differences in
Strengths and limitations of this study

- This is a longitudinal, school-based cohort study to assess the incidence and prevalence of myopia among school-age children in both urban and rural settings.

- Large sample size and the representative study population.

- Light exposure and daily activities will be measured using wearable devices in a random subsample of the cohort.

- A myopia prediction model will be established using a machine learning algorithm based on the collected data.

the prevalence of myopia may provide new clues for the intervention of myopia.

There is now considerable evidence that increased time outdoors reduces the onset of myopia, ranging from cross-sectional and longitudinal epidemiological studies, ${ }^{6} 7$ through to randomised clinical trials. ${ }^{8-10}$ In addition, a proposed mechanism based on exposures to brighter light outdoors has been demonstrated in animal models. ${ }^{11-14}$ However, not all studies have reported positive effects. ${ }^{15}$ Possible factors that could obscure a real association include lack of variation in time outdoors, or in prevalence and incidence of myopia, poor measurement of time outdoors ${ }^{16-18}$ and myopia and small sample size.

As urban and rural environments differ in many aspects, this may be complicated by confounding factors. Although population density can partly explain the urbanrural differences in myopia, it is mixed with factors such as living environment, behavioral patterns and visual stimulation. ${ }^{219}$ The effects of urbanisation may be confounded by factors such as education, socioeconomic status and outdoor exposure. It is still not entirely clear 
whether and how these factors in an urban environment could have an impact on the development and progression of myopia. ${ }^{20}$

We, therefore, intend to conduct a school-based cohort study in both urban and rural settings to identify the incidence, prevalence, and environmental risk factors of myopia among school-aged children. All participants will be enrolled from primary schools in Zhaoqing city, China and followed up for 3 years. Our study design overcomes the above-mentioned factors by using the gold standard of cycloplegic refraction and objective measures of time outdoors on a subsample, on a large sample size recruited from a location that covers urban and rural areas where preliminary data indicate that the prevalence and incidence of myopia vary significantly.

\section{METHODS AND ANALYSIS}

This study was registered on Clinicaltrials.gov. It is estimated that the study dates would be from 14 December 2019 to 2 February 2023.

\section{Objective}

The purpose of this study is to identify the incidence, prevalence, and risk factors of myopia among school-age children in urban and rural regions of southern China.

\section{Study design}

We will conduct a 3-year, school-based, longitudinal cohort study. This study adheres to the Declaration of Helsinki and ethics approval will be obtained from the Zhongshan Ophthalmic Center Institutional Review Board.

\section{Eligibility criteria}

Inclusion criteria

- All first-grade students in urban (10 primary schools) and rural regions (10 primary schools), Zhaoqing city.

Exclusion Criteria

- No.

\section{Study setting and participants}

Zhaoqing is one of the major cities in Guangdong province and has a population of 4084600 in 2016. Zhaoqing is chosen because of its relatively stable population covering a broad socioeconomic spectrum. To determine how the environment affects the onset of myopia, firstgrade students aged $6-7$ will be recruited from both urban (Duanzhou District) and rural regions (Huaiji County). This period precedes the development of myopia in most children. The results on this sample will be compared with those from previous studies (eg, the Sydney Myopia Study). ${ }^{17}$ Table 1 shows the sociodemographic variables in different counties of Zhaoqing city. Satellite images for the Huaiji County and Duanzhou District are shown in figure 1.

\section{Recruitment}

A database containing the annual examination result of UCVA of students was provided by Zhaoqing Education Bureau. There are 25 primary schools in Duanzhou District and 30 in Huaiji County. Given that most of the primary schools have more than two classes in each grade, we will exclude schools with less than two classes to keep an equal number of participants in each school. To ensure that all schools will be represented in the selected sample, all primary schools in Duanzhou District and Huaiji County will be stratified into five strata, respectively, based on previous results of visual acuity (UCVA $\geq 20 / 25)$. We will randomly select two schools from each stratum for our study. In total, 10 schools from Duanzhou District and 10 from Huaiji County will be involved. Independent researchers will complete the randomisation process and communicate the results to the research team. After that, the research team will contact schools to recruit.

\section{Sample size}

The average size of each cluster is about 200 persons, that is, about the number of students in the first grade of each school. Assuming the intraclass correlation coefficient

Table 1 Sociodemographic data in Zhaoqing city*

\begin{tabular}{llccc}
\hline County & Number of inhabitants (thousands) & $\begin{array}{l}\text { Land area } \\
\text { (sq.km) }\end{array}$ & $\begin{array}{l}\text { Population density (inhabitant per } \\
\text { sq.km) }\end{array}$ & $\begin{array}{l}\text { GDP in 2019 } \\
\text { (billion RMB) }\end{array}$ \\
\hline Huaiji† & 860 & 3573 & 241 & 23.0 \\
Gaoyao & 800 & 2071 & 386 & 42.0 \\
Duanzhou† & 510 & 152 & 3355 & 42.0 \\
Sihui & 500 & 1258 & 397 & 58.7 \\
Guangning & 450 & 2459 & 183 & 15.9 \\
Fengkai & 420 & 2723 & 154 & 15.0 \\
Deqing & 360 & 2258 & 159 & 15.0 \\
Dinghu & 190 & 512 & 371 & 12.6 \\
\hline
\end{tabular}

*Data are available from The Guangdong Statistical Yearbook 2020.

†Huaiji and Duanzhou districts will be selected as the rural and urban study areas, respectively.

GDP, gross domestic product. 


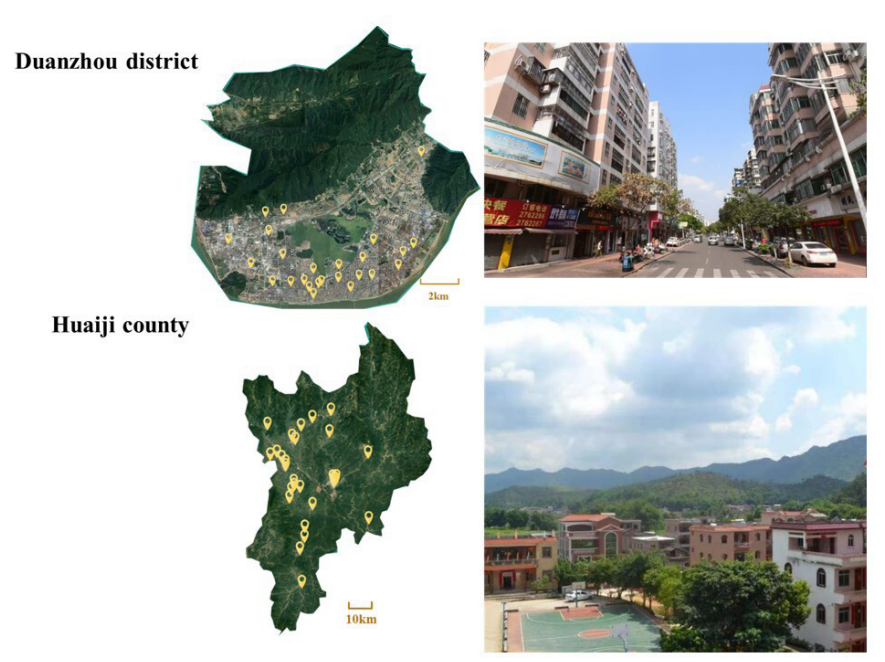

Figure 1 Satellite images for the Huaiji County and Duanzhou District. Most of the Duanzhou District (satellite image scale: 1:53 858) are covered by residential buildings, whereas Huaiji County (satellite image scale: 1:433 701) is mainly covered in greenery with mountains. Satellite images are available from http://wwwbigemapcom/source/tree/satel$244 \mathrm{html}$

within school is 0.015 with considering the variability of cluster sizes. Based on an cumulative incidence of about $35 \%$ of myopia for urban students ${ }^{11}$ and $25 \%$ for rural students, ${ }^{21}$ an estimated $10 \%$ loss to follow-up rate due to transfer or drop-out is included, for a total of about 20 schools consisting of 10 for the urban region and 10 for the rural region, which will provide $90 \%$ power at a twosided significant level of 0.05 to detect an approximately $10 \%$ difference in the cumulative incidence of myopia between urban and rural students. The sample size is calculated by PASS V.16.0 (NCSS, LLC, USA).

\section{Outcome measures \\ Primary outcome}

The primary outcome is incident myopia, defined as myopia detected during follow-up among those without myopia at baseline. Myopia in this study is defined as either eye's spherical equivalent refractive (SER) error (sphere $+1 / 2$ cylinder) of at least -0.5 diopters (D). However, we would also use the right eye data for the definition of myopia in some of the statistical analyses to ensure that our data could be comparable to the previous publications ${ }^{11}$ that used 'right eye data' in similar regions in Southern China.

\section{Secondary outcomes}

1. Prevalence of myopia (time frame: baseline) Myopia is defined as any eye's SER (sphere $+1 / 2$ cylinder) of at least -0.5 diopters (D).

2. Change in axial length (time frame: baseline, 3 years) Axial length will be measured with a non-contact optical device.

3. Prevalence of amblyopia, strabismus, and other ocular abnormalities (time frame: baseline)
Cover-uncover test will be performed to detect strabismus. Any ocular abnormalities, including corneal opacities, lens opacities and retinal diseases, will be recorded based on slit lamp, direct ophthalmoscopic, and/or mobile phone video examination. Participants with an UCVA $6 / 7.5$ or worse with undergo subjective refraction to identify amblyopia.

4. Area under the receiver operating characteristic (ROC) curve of the machine learning algorithm for the prediction of incident myopia (time frame: last year)

The investigators will estimate the area under the ROC curve of the machine learning algorithm for the prediction of incident myopia.

5. Sensitivity and specificity of the machine learning algorithm for the prediction of incident myopia (time frame: last year)

The investigators will estimate the sensitivity and specificity of the machine learning algorithm for the prediction of incident myopia.

6. Area under the ROC curve of the machine learning algorithm for the prediction of a fast progressing myope (time frame: last year)

The investigators will estimate the area under the ROC curve of the machine learning algorithm for the prediction of fast progressing myope (a change in SER of $0.75 \mathrm{D}$ or more per year).

7. Sensitivity and specificity of the machine learning algorithm for the prediction of fast progressing myope (time frame: last year)

The investigators will estimate the sensitivity and specificity of the machine learning algorithm for the prediction of fast progressing myope. Cycloplegic spherical refraction changes measured by an autorefractometer will be used as the indicator of myopia progression.

8. Area under the ROC curve of the diagnostic algorithm in identifying abnormal vision screening result (time frame: baseline)

The investigators will estimate the area under the ROC curve of the diagnostic algorithm in identifying abnormal vision screening result (eg, abnormal eye lid, abnormal cornea and strabismus detected with mobile devices).

9. Sensitivity and specificity of the diagnostic algorithm in identifying abnormal vision screening result (time frame: baseline)

The investigators will estimate the sensitivity and specificity of the diagnostic algorithm in identifying abnormal vision screening result (eg, abnormal eyelid, abnormal cornea and strabismus detected with mobile devices).

10. Postvision screening referral uptake (time frame: baseline)

Any referral uptake will be confirmed by telephone follow-up. 


\section{Examinations}

The children's height will be measured using a wallmounted scale reading device. Weight will be recorded in kilogram to one decimal place. Ophthalmic examinations will include visual acuity, cover test, and ocular dominance, non-cycloplegic autorefraction, cycloplegia, ocular biometric measurements, cycloplegic autorefraction, subjective refraction and anterior and posterior segment examination. Any ocular abnormalities, including corneal opacities, lens opacities and retinal diseases, will be recorded based on slit lamp and direct ophthalmoscopic examination.

\section{Questionnaires}

A family questionnaire ${ }^{7}$ regarding educational attainment, occupations, refractive status and history of eye disease will be administered only at baseline. The grade leader teacher or headteacher of each class will be asked to complete a class curriculum questionnaire ${ }^{7}$ about the curriculum schedule (eg, school days, school holidays). Sleep quality and quantity will be measured with the Patient-Reported Outcomes Information System (PROMIS) -Sleep Disorder questionnaire. ${ }^{22}$ Time (hour) spent in different activities (separately for outdoor activities, near work activities, screen time (separately for smartphone, tablet, TV, computer, games console)) will be measured with a Self-reported Previous Day Activity Recall Questionnaire. These two questionnaires will be collected at baseline.

\section{Subsample study}

1. Spectacle-frame mounted distance sensor and eye-tracking.

Time (hour) spent in different activities will be measured with an eye tracker (time frame: participants will be asked to meet the study investigators at school, and they will be provided with a spectacle-frame eye tracker to be worn for 2 hours or more during the daytime and 2 hours or more during the night time on the next day.

2. Wearable activity tracker

Physical activity and light intensity will be measured with a wearable activity tracker (time frame: baseline). On each occasion, participants will be asked to meet the study investigators at school, and they will be provided with a wearable activity tracker to be worn on the wrist for 24 hours on the next day.

3. Optical coherence tomography (OCT)

An OCT Instrument (Moptim Mocean-3500) will be used to obtain high-resolution macular images which could show choroid thickness. [Time frame: baseline].

\section{Workflow}

The workflow will follow the order as above mentioned, starting from body measurement and ending with a direct ophthalmoscopic examination (figures 2 and 3). Participants will be followed up to maximise participation in the eye examinations at baseline and annually at years 1 ,

Primary schools in Duanzhou and Huaiji

\section{Enrollment and informed consent}

10 rural schools in Huaiji and 10 urban schools in Duanzhou
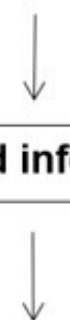

\section{Baseline examinations}

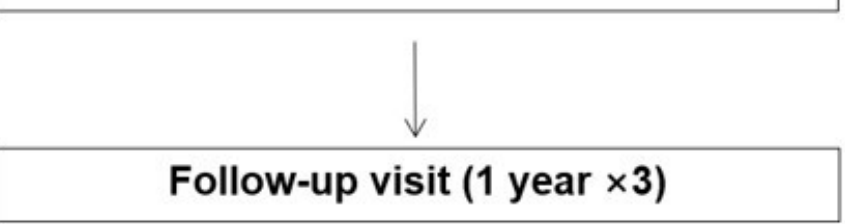

Figure 2 Flowchart of the study.

2, and 3, with the same examiner using the same set of instruments with regular calibration.

\section{Data management}

The clinical examiner will regularly check the informed consent and eligibility for each participant to guarantee

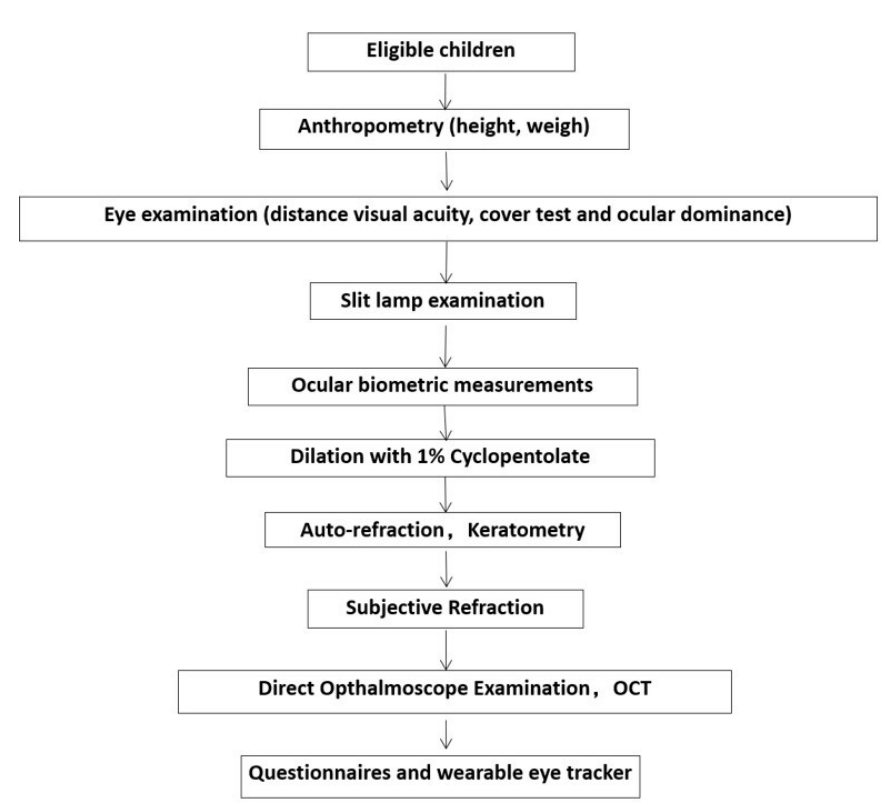

Figure 3 Flowchart of field examinations. OCT, optical coherence tomography. 
that all case report form (CRF) forms are correct and in accordance with the original data. All errors and omissions will be noted, and where possible corrected. The examiner will also ensure every participant's withdrawal and loss are recorded and explained in CRF, and all adverse events are recorded.

Results of eye examinations will be recorded on paper, except for those obtained from electronic questionnaires and ocular biometry measurements. To ensure data accuracy, data will be entered by two independent personnel for further data checking. After double data entry, we will select $5 \%$ of cases at random to identify any inconsistency between the original document and the final electronic record.

Examination forms and questionnaires will be reviewed and completed before data entry at the Zhongshan Ophthalmic Center. Range of data, frequency distribution, and the consistency among related measurements are checked using data cleaning programs. The right eye of each student will be included in data analysis. If data for the right eye are not available, the left eye will be included in the analysis.

\section{Statistical analysis plan}

Statistical analysis will be performed using Stata V.16 software (StataCorp, College Station Texas, USA). The distribution of baseline characteristics will be reported by use of mean (SD) or median (IQR) for continuous variables and frequency (percentage) for categorical variables. Baseline comparisons between the urban groups and the rural groups will be performed by using two-sample t-test for continuous variables, and $\chi^{2}$ test for categorical variables. Mann-Whitney test will be used when dependent variables are not normally distributed. The normality of the continuous data distribution will be checked by Shapiro-Wilk normality test and histogram. The differences and 95\% CI between urban and rural students groups in the mean change of SE andaxial length (AL) will be calculated using the linear regression analysis. The cumulative incidence of myopia and its associated risk factors will be estimated using univariable and multivariable logistic regression models. All variables with $\mathrm{p}<0.05$ in the univariable regression analysis will be included in the multivariable regression model. All comparisons and regression analysis will be adjusted for the cluster effect between eyes within the same subject. A two-sided $\mathrm{p}<0.05$ will be considered statistically significant.

The data set will include all participants who complete 3 years of follow-up and examination. We divide the data into training sets, cross-validation sets, and test sets in a ratio of 5:2:3. Predictors include a set of baseline data including refractive status, biological parameters, demographic information, family information, daily activity, sleep and screen time. Based on these predictors, we aim to develop a machine learning algorithm to predict participants' SE and to predict whether they have myopia after 3 years. The performance evaluation of regression algorithm for predicting target $\mathrm{SE}$ includes three evaluation indexes: coefficient of determination (R2), root mean square error and mean absolute error. The rROC curves and area under the curve values will be used to assess myopia classification performance. ${ }^{23}$

\section{Study monitoring}

At the start of the project, the Case Report Form, workflow, quality control procedures, electronic questionnaire, data entry methods and follow-up management will be detailed in the training courses.

\section{Patient and public involvement}

Participants are not invited to comment on the study design and aim or to interpret the findings, contribute to the writing or editing of this document.

\section{Ethics and dissemination}

This project has obtained ethics approval from the ethics committee of the Zhongshan Ophthalmic Center (number: 2019KYPJ171). Parents (or other authorised surrogates) of participants give informed consent before taking part. The content of scientific research complies with the principles of the Declaration of Helsinki and its regulations applicable to research and human participants. The study investigators should protect participants' privacy and are responsible for the confidentiality of all private information. The principal investigator is responsible for informing the ethics committee of any amendments to the protocol. The baseline and annual results will be published in peer-reviewed journals.

Clinical records and data sets will be kept at the Zhongshan Ophthalmic Center. Any public report of the results will not disclose personal information. Data will be deidentified before being passed to the study statisticians. Data will be kept in strict confidence and will only be assessed by the study investigators and authorised personnel. All data documents will be password protected, stored on a secure server, kept for at least 10 years and destroyed if no longer retention is required. If the project is modified, it will be exposed on Clinicaltrials.gov.

\section{DISCUSSION}

It is estimated that the financial burden of myopiarelated visual impairment reached US\$244 billion in 2015 globally. ${ }^{24}$ Since myopia generally develops during the school years, and tends to stabilise in adulthood, interventions to control the development of myopia need to be implemented during the school years. ${ }^{1}$ Increasing time spent outdoors is the main protective method to prevent myopia. ${ }^{25}$ The efficacy of optical interventions (spectacles, soft lenses and, orthokeratology lenses) ${ }^{26} 27$ and pharmacological interventions (low-dose atropine) ${ }^{28}$ have been shown to be effective in slowing the progression of myopia. It is, therefore, imperative to explore the risk factors and predict the development of myopia among school-age children. In this current study, we will examine the association between environmental factors 
and myopia in urban and rural settings using subjective and objective data collection methods. Also, we will use neural learning algorithm to integrate ocular biological characteristics and environmental variables to establish an artificial intelligence model. In this way, we can effectively predict the occurrence and progression of myopia and lay the foundation for individualised myopia prevention and control in the future.

\section{Author affiliations}

${ }^{1}$ State Key Laboratory of Ophthalmology, Zhongshan Ophthalmic Center, Sun Yatsen University, Guangzhou, China

${ }^{2}$ Guangzhou Regenerative Medicine and Health Guangdong Laboratory, Guangzhou, China

${ }^{3}$ Sun Yat-Sen University Zhongshan Ophthalmic Center, Guangzhou, Guangdong, China

${ }^{4}$ Australian National University Research School of Biological Sciences-RSBS, Canberra, Australian Capital Territory, Australia

Acknowledgements We are grateful to Zhaoging Education Bureau for providing eye examination database of students and all involved staff for their cooperation.

Contributors MI, YL and YiZ conceived and designed the study. XC, GY, YuZ, YaZ and YiZ wrote the draft. YaZ, MI, XL and YiZ revised draft. LJ will lead the statistical analysis. YiZ and YuZ will oversee data acquisition and implementation on site. All authors reviewed and approved the final manuscript.

Funding This study was supported by the Construction Project of High-Level Hospitals in Guangdong Province (303020107; 303010303058); National Natural Science Foundation of China (81530028; 81721003); Clinical Innovation Research Program of Guangzhou Regenerative Medicine and Health Guangdong Laboratory (2018GZR0201001); Research Units of Ocular Development and Regeneration, Chinese Academy of Medical Sciences (2019-I2M-5-005); Local Innovative and Research Teams Project of Guangdong Pearl River Talents Program; the State Key Laboratory of Ophthalmology, Zhongshan Ophthalmic Center, Sun Yat-sen University.

Map disclaimer The depiction of boundaries on this map does not imply the expression of any opinion whatsoever on the part of BMJ (or any member of its group) concerning the legal status of any country, territory, jurisdiction or area or of its authorities. This map is provided without any warranty of any kind, either express or implied.

Competing interests MI has received personal support from Essilor. The other authors have declared that no competing interests exist.

Patient and public involvement Patients and/or the public were not involved in the design, or conduct, or reporting, or dissemination plans of this research.

Patient consent for publication Not applicable.

Provenance and peer review Not commissioned; externally peer reviewed.

Open access This is an open access article distributed in accordance with the Creative Commons Attribution Non Commercial (CC BY-NC 4.0) license, which permits others to distribute, remix, adapt, build upon this work non-commercially, and license their derivative works on different terms, provided the original work is properly cited, appropriate credit is given, any changes made indicated, and the use is non-commercial. See: http://creativecommons.org/licenses/by-nc/4.0/.

\section{ORCID iD}

Yingfeng Zheng http://orcid.org/0000-0002-0914-7864

\section{REFERENCES}

1 Morgan IG, Ohno-Matsui K, Saw S-M. Myopia. Lancet 2012;379:1739-48.

2 Ip JM, Rose KA, Morgan IG, et al. Myopia and the urban environment: findings in a sample of 12-year-old Australian school children. Invest Ophthalmol Vis Sci 2008;49:3858-63.
3 He M, Huang W, Zheng Y, et al. Refractive error and visual impairment in school children in rural southern China. Ophthalmology 2007;114:374-82.

4 Pan C-W, Wu R-K, Li J, et al. Low prevalence of myopia among school children in rural China. BMC Ophthalmol 2018;18:140.

5 Dandona R, Dandona L, Srinivas M, et al. Refractive error in children in a rural population in India. Invest Ophthalmol Vis Sci 2002;43:615-22.

6 Li S-M, Liu L-R, Li S-Y, SM L, SY L, et al. Design, methodology and baseline data of a school-based cohort study in central China: the anyang childhood eye study. Ophthalmic Epidemiol 2013;20:348-59.

7 Ojaimi E, Rose KA, Smith W, et al. Methods for a population-based study of myopia and other eye conditions in school children: the Sydney myopia study. Ophthalmic Epidemiol 2005;12:59-69.

8 Negrel AD, Maul E, Pokharel GP, et al. Refractive error study in children: sampling and measurement methods for a multi-country survey. Am J Ophthalmol 2000;129:421-6.

9 Rose KA, Morgan IG, Ip J, et al. Outdoor activity reduces the prevalence of myopia in children. Ophthalmology 2008;115:1279-85.

10 Read SA, Collins MJ, Vincent SJ. Light exposure and eye growth in childhood. Invest Ophthalmol Vis Sci 2015;56:6779-87.

$11 \mathrm{He} \mathrm{M}$, Xiang F, Zeng Y, et al. Effect of time spent outdoors at school on the development of myopia among children in China: a randomized clinical trial. JAMA 2015;314:1142-8.

12 Wu P-C, Chen C-T, Lin K-K, et al. Myopia prevention and outdoor light intensity in a school-based cluster randomized trial. Ophthalmology 2018;125:1239-50.

13 Zadnik K, Mutti DO. Outdoor activity protects against childhood myopia-let the sun shine in. JAMA Pediatr 2019;173:415-6.

14 Ashby R, Ohlendorf A, Schaeffel F. The effect of ambient illuminance on the development of deprivation myopia in chicks. Invest Ophthalmol Vis Sci 2009;50:5348-54.

15 Ashby RS, Schaeffel F. The effect of bright light on lens compensation in chicks. Invest Ophthalmol Vis Sci 2010;51:5247-53.

16 Smith EL, Hung L-F, Huang J. Protective effects of high ambient lighting on the development of form-deprivation myopia in rhesus monkeys. Invest Ophthalmol Vis Sci 2012;53:421-8.

17 Norton TT, Amedo AO, Siegwart JT. Darkness causes myopia in visually experienced tree shrews. Invest Ophthalmol Vis Sci 2006;47:4700-7.

18 Ngo CS, Pan C-W, Finkelstein EA, et al. A cluster randomised controlled trial evaluating an incentive-based outdoor physical activity programme to increase outdoor time and prevent myopia in children. Ophthalmic Physiol Opt 2014;34:362-8.

19 Zhang M, Li L, Chen L, et al. Population density and refractive error among Chinese children. Invest Ophthalmol Vis Sci 2010;51:4969-76.

$20 \mathrm{He}$ M, Zheng Y, Xiang F. Prevalence of myopia in urban and rural children in mainland China. Optom Vis Sci 2009;86:40-4.

21 Guo Y, Liu L, Lv Y, et al. Outdoor jogging and myopia progression in school children from rural Beijing: the Beijing children eye study. Trans/ Vis Sci Technol 2019;8:2.

22 Buysse DJ, Yu L, Moul DE, et al. Development and validation of patient-reported outcome measures for sleep disturbance and sleeprelated impairments. Sleep 2010;33:781-92.

23 Lin H, Long E, Ding X, et al. Prediction of myopia development among Chinese school-aged children using refraction data from electronic medical records: a retrospective, multicentre machine learning study. PLoS Med 2018;15:e1002674.

24 Naidoo KS, Fricke TR, Frick KD, et al. Potential lost productivity resulting from the global burden of myopia: systematic review, metaanalysis, and modeling. Ophthalmology 2019;126:338-46.

25 Sherwin JC, Reacher MH, Keogh RH, et al. The association between time spent outdoors and myopia in children and adolescents: a systematic review and meta-analysis. Ophthalmology 2012:119:2141-51.

26 Walline JJ, Lindsley K, Vedula SS, et al. Interventions to slow progression of myopia in children. Cochrane Database Syst Rev 2011;12:Cd004916.

27 Huang J, Wen D, Wang Q, et al. Efficacy comparison of 16 interventions for myopia control in children: a network meta-analysis. Ophthalmology 2016;123:697-708.

28 Gong Q, Janowski M, Luo M, et al. Efficacy and adverse effects of atropine in childhood myopia: a meta-analysis. JAMA Ophthalmol 2017;135:624-30. 